\title{
Three-year outcomes of a phase II study of adjuvant chemotherapy with S-1 plus docetaxel for stage III gastric cancer after curative D2 gastrectomy
}

\author{
Kazumasa Fujitani - Shigeyuki Tamura Yutaka Kimura • Takeshi Tsuji · Jin Matsuyama • \\ Shohei Iijima · Hiroshi Imamura · Kentaro Inoue - Kenji Kobayashi · Yukinori Kurokawa • \\ Toshio Shimokawa $\cdot$ Toshimasa Tsujinaka $\cdot$ Hiroshi Furukawa
}

Received: 4 February 2013/Accepted: 15 May 2013/Published online: 5 June 2013

(C) The International Gastric Cancer Association and The Japanese Gastric Cancer Association 2013

\begin{abstract}
Background We have previously reported the superior feasibility and safety of adjuvant S-1 plus docetaxel in patients with stage III gastric cancer during a prospective phase II study. We report 3-year follow-up data on patients enrolled in this study.

Patients and methods Fifty-three patients with histologically confirmed stage III gastric cancer who underwent gastrectomy with D2 lymphadenectomy were enrolled into this study. They received oral S-1 $\left(80 \mathrm{mg} / \mathrm{m}^{2} /\right.$ day $)$ for 2 consecutive weeks and intravenous docetaxel $\left(40 \mathrm{mg} / \mathrm{m}^{2}\right)$ on
\end{abstract}

On the behalf of the Osaka Gastrointestinal Cancer Chemotherapy Study Group.

K. Fujitani $(\bowtie) \cdot T$. Tsujinaka

Department of Surgery, Osaka National Hospital,

2-1-14 Hoenzaka, Chuo-ku, Osaka 540-0006, Japan

e-mail: fujitani@onh.go.jp

S. Tamura

Department of Surgery, Kansai Rosai Hospital, Hyogo, Japan

\section{Y. Kimura}

Department of Surgery, NTT West Osaka Hospital, Osaka, Japan

T. Tsuji

Department of Surgery, Wakayama Rosai Hospital,

Wakayama, Japan

J. Matsuyama

Department of Surgery, Yao Municipal Hospital, Osaka, Japan

S. Iijima

Department of Surgery, Minoh City Hospital, Osaka, Japan

H. Imamura

Department of Surgery, Sakai Municipal Hospital,

Osaka, Japan day 1 , repeated every 3 weeks (one cycle). Treatment was initiated within 45 days after surgery and repeated for four cycles, followed by S-1 monotherapy (4 weeks on, 2 weeks off) until 1 year after surgery. Three-year overall survival (OS) and disease-free survival (DFS) were evaluated.

Results The OS rate at 3 years was $78.4 \%$ [95\% confidence interval (CI), 67.9-90.6\%] and the DFS rate at 3 years was $66.2 \%$ (95\% CI, 54.4-80.7\%). Subgroup analyses according to disease stage showed a 3-year OS and DFS rate of $85.7 \%$ (95\% CI, 74.9-98.1\%) and $70.8 \%$ (95\% CI, 57.1-87.8\%) for stage IIIA, and $62.5 \%$ $(95 \%$ CI, $42.8-91.4 \%)$ and $56.2 \% \quad(95 \%$ CI, $36.5-86.7 \%$ ) for stage IIIB, respectively.

Conclusions On the basis of 3-year follow-up data, postoperative adjuvant therapy with S-1 plus docetaxel

K. Inoue

Department of Surgery, Kansai Medical University, Osaka, Japan

K. Kobayashi

Department of Surgery, Kinki Central Hospital, Hyogo, Japan

Y. Kurokawa

Department of Surgery, Osaka University Medical School,

Osaka, Japan

T. Shimokawa

Graduate School of Medicine and Engineering,

Yamanashi University, Yamanashi, Japan

H. Furukawa

Department of Surgery, Kinki University Medical School,

Osaka, Japan 
yielded promising OS and DFS in stage IIIA gastric cancer patients who had undergone D2 gastrectomy. We believe that this regimen is a candidate for future phase III trials studying the optimal adjuvant chemotherapy regimen for stage III gastric cancer.

Keywords Adjuvant chemotherapy - Docetaxel · Gastric cancer $\cdot$ Three-year survival $\cdot \mathrm{S}-1$

\section{Introduction}

Although several meta-analyses have suggested that adjuvant chemotherapy provides a survival benefit for gastric cancer [1-7], efficacy has been established for only a few treatments in large clinical trials. Postoperative radiotherapy with 5-FU plus leucovorin has become a standard adjuvant regimen in the US [8], and the perioperative triplet regimen of epirubicin, cisplatin, and 5-FU is standard in the UK [9]. In Japan, adjuvant chemotherapy with $S-1$ is the current standard of care after curative gastrectomy with D2 lymphadenectomy (D2 gastrectomy) for histologically confirmed stage II/III disease [10]. Recently, 5-year followup data have been reported [11]. The S-1 group had a 5-year overall survival (OS) rate of $71.7 \%$, compared with $61.1 \%$ in the surgery-alone group, corresponding to a $33 \%$ reduced risk of death [hazard ratio (HR), 0.669; $95 \%$ confidence interval (CI), 0.540-0.828]. However, approximately $35 \%$ of patients still develop recurrence despite adjuvant $\mathrm{S}-1$, and subgroup analyses have suggested $\mathrm{S}-1$ is less efficacious for stage IIIB gastric cancer (HR, 0.791; $95 \%$ CI, 0.520-1.205), in contrast to a clear survival benefit of S-1 demonstrated for stage II and IIIA disease [11].

Several attempts have been made to improve the efficacy of adjuvant S-1 chemotherapy. For example, Takahari et al. [12] have evaluated the feasibility of three cycles of S-1 plus cisplatin with subsequent S-1 monotherapy until 1 year after surgery for stage III gastric cancer. We have previously demonstrated that four cycles of S-1 plus docetaxel followed by S-1 monotherapy up to 1 year after surgery is a feasible regimen with moderate toxicity when used as adjuvant chemotherapy in patients with stage III gastric cancer after curative D2 gastrectomy [13].

The aim of this study was to evaluate the efficacy of S-1 plus docetaxel adjuvant chemotherapy for stage III gastric cancer in terms of survival to determine if this regimen is a potential candidate for the next adjuvant phase III chemotherapy trial.

\section{Patients and methods}

This study was conducted in accordance with the international ethical recommendations stated in the Declaration of Helsinki. The protocol was approved by the institutional review and ethics board of each participating hospital and was registered in the University Hospital Medical Information Network (UMIN) database (ID 000000714). Written informed consent was obtained from all patients.

\section{Eligibility}

Eligibility criteria were as follows: histopathologically confirmed stage IIIA or IIIB gastric cancer; R0 resection (with no tumor cells at the margin) with D2 or more extensive lymph node dissection; no evidence of hepatic, peritoneal, or distant metastasis; no tumor cells on peritoneal lavage cytology; age 20-80 years; Eastern Cooperative Oncology Group performance status $0-1$; no previous treatment for cancer except for initial gastric resection of the primary lesion; adequate organ function; absence of other severe medical conditions; and absence of synchronous or metachronous malignancies. Tumor stage classification and D classification were in accordance with the Japanese Classification of Gastric Carcinoma (second English edition) [14]. Patients were enrolled within 6 weeks after surgery by means of facsimile.

\section{Treatment}

Patients were given S-1 in a daily dose of 80,100 , or $120 \mathrm{mg}$ in two divided doses based on body surface area for 2 consecutive weeks with intravenous docetaxel $\left(40 \mathrm{mg} / \mathrm{m}^{2}\right)$ on day 1 , repeated every 3 weeks (one cycle). The treatment was started within 45 days after surgery and repeated for four cycles. After four cycles of S-1 plus docetaxel, S-1 monotherapy (4 weeks on, 2 weeks off) was continued until 1 year after surgery. The criteria for dose reduction resulting from toxicity have been described elsewhere [13].

\section{Follow-up}

Patients underwent hematological tests and assessments of clinical symptoms at least once during each cycle of combination chemotherapy with S-1 plus docetaxel and at 6-week intervals during S-1 monotherapy. From the second year onward, all patients were followed up at least every 3 months. Relapse was confirmed by imaging studies, including ultrasonography, computed tomography, and gastrointestinal endoscopy. Patients underwent abdominal computed tomography at intervals of 6 months or less for the first 3 years after surgery, and at 1-year intervals thereafter until 5 years after surgery, and also received gastrointestinal endoscopy at 1-year intervals. All patients were followed up for at least 3 years from the date of 
treatment initiation or until death. None was lost to followup.

\section{Statistical analysis}

The sample size was calculated with an expected feasibility rate of $75 \%$ and a threshold feasibility rate of $50 \%$ for four cycles of treatments with S-1 plus docetaxel based on a two-sided alpha level of 0.05 and statistical power of $90 \%$. The planned sample size was 50 patients, allowing for a $20 \%$ dropout rate. OS and disease-free survival (DFS) for up to 3 years from the date of treatment initiation were estimated for all patients and analyzed according to disease stage. OS was defined as the time from the date of treatment initiation to the date of death from any cause or last follow-up. DFS was defined as the time from the date of treatment initiation to the date when recurrence or a second malignancy was confirmed, death from any cause, or last follow-up, whichever came first. The survival curves were estimated using the Kaplan-Meier method, and the $95 \%$ CIs for survival rate were estimated using Greenwood's formula. All statistical analyses were done with S-plus version 8.1 J (Mathematical Systems).

\section{Results}

Patients

Between May 2007 and August 2008, 53 patients (42 men and 11 women) with a median age of 65 years (range, 43-78) were enrolled from 13 institutions. Based on the Japanese classification criteria, 35 patients $(66.0 \%)$ had stage IIIA disease and 16 patients $(30.2 \%)$ had stage IIIB disease. There was 1 patient with stage II (T1N2) and IV (positive tumor cells on peritoneal lavage cytology) disease each. Patient characteristics are listed in Table 1. Details of feasibility, safety, and reasons for treatment discontinuation were described previously [13]. In brief, the completion rate of the planned four cycles of S-1 plus docetaxel was $79.2 \%$ (95\% CI, 65.9-89.2\%), whereas the completion rate of four cycles of S-1 plus docetaxel and subsequent S-1 monotherapy for 1 year was $64.2 \%$ (95 \% CI, 49.8-76.9\%). The relative dose intensity of $\mathrm{S}-1$ and docetaxel for four cycles of chemotherapy were $79.6 \%$ and $87.8 \%$, respectively.

\section{OS and DFS}

Survival analyses were performed on 51 patients, excluding the 2 patients whose disease stage did not fulfill the inclusion criteria. Fifteen patients died, of whom 13 died of relapsed disease and 2 of other causes such as systemic infection and
Table 1 Patient characteristics

\begin{tabular}{|c|c|}
\hline & Patients $(n=53)$ \\
\hline Age (years), median (range) & $65(43-78)$ \\
\hline Male:female & $42: 11$ \\
\hline \multicolumn{2}{|l|}{ ECOG PS } \\
\hline 0 & 31 \\
\hline 1 & 22 \\
\hline \multicolumn{2}{|l|}{ Pathological type } \\
\hline Intestinal & 24 \\
\hline Diffuse & 29 \\
\hline \multicolumn{2}{|l|}{ Stage $^{\mathrm{a}}$} \\
\hline II & 1 \\
\hline IIIA & 35 \\
\hline IIIB & 16 \\
\hline IV & 1 \\
\hline \multicolumn{2}{|l|}{$\mathrm{T}$ stage $^{\mathrm{b}}$} \\
\hline pT1 & 1 \\
\hline pT2 & 20 \\
\hline pT3 & 30 \\
\hline pT4 & 2 \\
\hline \multicolumn{2}{|l|}{$\mathrm{N}$ stage $^{\mathrm{b}}$} \\
\hline pNO & 1 \\
\hline $\mathrm{pN} 1$ & 26 \\
\hline $\mathrm{pN} 2$ & 19 \\
\hline $\mathrm{pN} 3$ & 7 \\
\hline \multicolumn{2}{|l|}{ M stage ${ }^{b}$} \\
\hline M0 & 52 \\
\hline M1 & 1 \\
\hline \multicolumn{2}{|l|}{ Stage $^{b}$} \\
\hline II & 11 \\
\hline IIIA & 24 \\
\hline IIIB & 9 \\
\hline IV & 9 \\
\hline
\end{tabular}

ECOG PS Eastern Cooperative Oncology Group performance status

a Japanese classification

b TNM classification by the 6 th edition

pulmonary emphysema. Thirty-three patients are alive without recurrence. In addition, 1 patient is alive with a second malignancy and 2 experienced recurrence. No treatment-related deaths occurred within 30 days after treatment completion. The 3-year OS and DFS rates for all eligible 51 patients were $78.4 \%$ (95\% CI, 67.9-90.6\%) and $66.2 \%$ (95\% CI, 54.4-80.7\%), respectively, as shown in Fig. 1a, b. Kaplan-Meier estimates of the 3-year OS and DFS are shown according to disease stage, with 3-year OS rates of $85.7 \%(95 \% \mathrm{CI}, 74.9-98.1 \%)$ and $62.5 \%(95 \%$ CI, 42.8-91.4\%) for stages IIIA and IIIB (Fig. 2a), respectively, and 3-year DFS rates of $70.8 \%(95 \% \mathrm{CI}$, $57.1-87.8 \%$ ) and $56.2 \%$ (95 \% CI, 36.5-86.7\%) for stages IIIA and IIIB (Fig. 2b), respectively. 

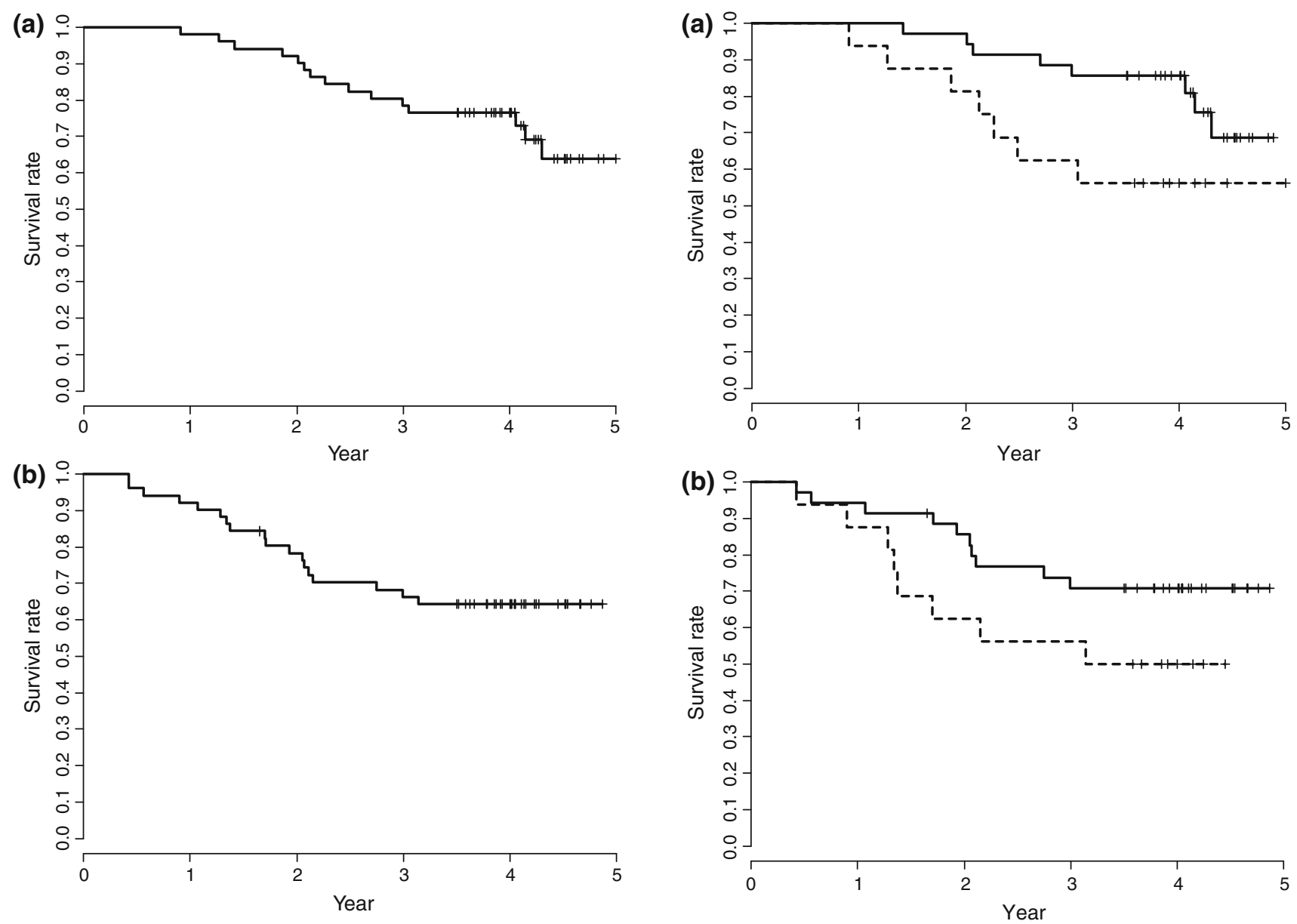

Fig. 1 a Kaplan-Meier estimates of 3-year overall survival for the eligible 51 patients. b Kaplan-Meier estimates of 3-year disease-free survival for the eligible 51 patients

Site of relapse

Fifteen patients had relapsed disease, involving nodal recurrence in 9 patients $(1$ cervical, 8 abdominal including paraaortic), hematogenous recurrence in 6 patients (5 liver, 1 spleen, 1 bone, 1 brain), and peritoneal dissemination in 4 patients.

\section{Discussion}

The mainstay of treatment for gastric cancer is surgery. However, in stage II and III disease, quite a few patients experience recurrence, even after curative resection. Adjuvant chemotherapy is used to prevent distant or local recurrence and improve survival. A recent meta-analysis by the Global Advanced/Adjuvant Stomach Tumor Research International Collaboration (GASTRIC) group [7] has shown that postoperative adjuvant chemotherapy is associated with an $18 \%$ risk reduction for both DFS (HR, 0.82; $95 \%$ CI, 0.75-0.90) and OS (HR, 0.82; $95 \%$ CI,

Fig. 2 a Kaplan-Meier estimates of 3-year overall survival for patients with stages IIIA and IIIB gastric cancer. Solid line, IIIA; dotted line, IIIB. b Kaplan-Meier estimates of 3-year disease-free survival for patients with stages IIIA and IIIB gastric cancer. Solid line, IIIA; dotted line, IIIB

0.76-0.90) compared with surgery alone in patients with resectable gastric cancer. One year of S-1 monotherapy after D2 gastrectomy has been established as the standard of care in Japan for patients with stage II or III gastric cancer, with a $33 \%$ reduced risk of death (HR, 0.669; $95 \%$ CI, 0.540-0.828) [11], which was comparable to the mortality risk reduction of $26 \%$ obtained by postoperative chemoradiotherapy in the US [8] or the $25 \%$ obtained by perioperative triplet chemotherapy in the UK [9]. However, approximately one-third of patients still relapse despite adjuvant treatment with $\mathrm{S}-1$, and subgroup analyses have shown insufficient efficacy of S-1 for stage IIIB disease (HR, 0.791; $95 \%$ CI, 0.520-1.205) [11], suggesting there remains some room for improvement.

To improve the efficacy of adjuvant chemotherapy, another agent, docetaxel or cisplatin, both of which have shown effective cytotoxicity against advanced gastric cancer in several randomized controlled trials [15-17], was investigated in combination with S-1 for feasibility in the 
adjuvant setting. Four cycles of S-1 plus docetaxel followed by S-1 monotherapy up to 1 year after curative D2 gastrectomy showed superior feasibility, with $79.2 \%$ (95\% CI, 65.9-89.2) feasibility for the four planned cycles of $\mathrm{S}-1$ plus docetaxel and good compliance with S-1 monotherapy at 12 months after surgery in $64.2 \%$ of patients with stage III gastric cancer [13]. Treatment compliance at 12 months was compatible with results of the ACTS-GC trial that established 1 year of adjuvant S-1 monotherapy as the Japanese standard of care [10]. Similarly, three cycles of S-1 plus cisplatin subsequent to the first cycle of S-1 monotherapy was shown to be feasible in $81 \%(95 \%$ CI, 65-92) of patients with stage III gastric cancer [12].

Because of its high feasibility, the survival data for postoperative adjuvant chemotherapy with S-1 plus docetaxel followed by S-1 monotherapy for up to 1 year after surgery are eagerly awaited, and this regimen is now considered a potential candidate for an experimental arm in the next adjuvant phase III trial. The 3-year OS and DFS rates for the 51 eligible patients were $78.4 \%(95 \% \mathrm{CI}$, $67.9-90.6 \%)$ and $66.2 \% \quad(95 \%$ CI, 54.4-80.7\%), respectively (Fig. 1a, b). Kaplan-Meier estimates of the 3 -year OS and DFS rates are shown according to disease stage, with 3-year OS rates of $85.7 \%(95 \% \mathrm{CI}$, $74.9-98.1 \%)$ and $62.5 \%(95 \% \mathrm{CI}, 42.8-91.4 \%)$ for stages IIIA and IIIB (Fig. 2a), and 3-year DFS rates of $70.8 \%(95 \% \mathrm{CI}, 57.1-87.8 \%)$ and $56.2 \%(95 \% \mathrm{CI}$, 36.5-86.7\%) for stages IIIA and IIIB (Fig. 2b), respectively. In comparison, patients receiving adjuvant S-1 monotherapy in the ACTS-GC trial had 3-year OS rates of $77.4 \%$ and $64.3 \%$ for stages IIIA and IIIB, respectively, and 3-year relapse-free survival (RFS) rates of $69.1 \%$ and $49.9 \%$ for stages IIIA and IIIB, respectively (Table 2) [10, 18]. We also evaluated the stage-specific 3-year OS and RFS according to the sixth edition of the International Union Against Cancer (UICC) TNM Classification of Malignant Tumours [19]. As shown in Table 2, for stage IIIA, 3-year OS and RFS rates according to UICC staging

Table 2 Comparison of results of the current study and the ACTSGC trial

\begin{tabular}{llllll}
\hline & \multicolumn{2}{l}{ 3-year DFS/RFS (\%) } & & \multicolumn{2}{l}{ 3-year OS (\%) } \\
\cline { 2 - 3 } \cline { 5 - 6 } & S-1 + DTX & ACTS-GC (S-1) & & S-1 + DTX & ACTS-GC (S-1) \\
\hline IIIA $^{\text {a }}$ & 70.8 & 69.1 & 85.7 & 77.4 \\
IIIB $^{\text {a }}$ & 56.2 & 49.9 & 62.5 & 64.3 \\
IIIA $^{\text {b }}$ & 79.2 & 69.1 & 95.8 & 77.8 \\
IIIB $^{\text {b }}$ & 44.4 & 44.8 & 55.6 & 66.6 \\
\hline
\end{tabular}

$D F S$ disease-free survival, $R F S$ relapse-free survival, $O S$ overall survival, $D T X$ docetaxel

a Japanese classification

b TNM classification by the 6th edition were $95.8 \%(95 \% \mathrm{CI}, 88.2-100 \%)$ and $79.2 \%$ (95\% CI, 64.5-97.2\%) with S-1 plus docetaxel, and for S-1 monotherapy they were $77.8 \%$ and $69.1 \%$ in the ACTS-GC trial [11]. Similarly, for stage IIIB, 3-year OS and RFS rates were $55.6 \%$ (95\% CI, 31.0-99.7\%) and $44.4 \%$ (95\% CI, 21.4-92.3\%) with S-1 plus docetaxel, compared to $66.6 \%$ and $44.8 \%$ with S-1 monotherapy, in the ACTSGC trial [11]. Based on these findings, adjuvant chemotherapy with four cycles of S-1 plus docetaxel followed by S-1 monotherapy until 1 year after surgery is expected to be more beneficial for UICC-TNM stage IIIA disease than S-1 monotherapy, but not for stage IIIB disease. However, the small sample size of each stage (UICC-TNM IIIA, $n=24$; IIIB, $n=9$ ) should be taken into careful consideration. Moreover, the recent CLASSIC trial [20] showed adjuvant chemotherapy with capecitabine plus oxaliplatin after D2 gastrectomy was associated with a survival benefit compared with surgery alone, with an improved 3-year DFS of $66 \%(95 \% \mathrm{CI}, 57-75)$ and $61 \%(95 \% \mathrm{CI}$, 48-73) for UICC-TNM stage IIIA and IIIB disease, respectively; this suggests that adjuvant $\mathrm{S}-1$ plus docetaxel may have a therapeutic benefit in stage IIIA gastric cancer. To further study its effect on stage IIIB disease, another phase II study of adjuvant S-1 with durable docetaxel for eight cycles is currently ongoing.

So far, 15 of 51 patients $(29.4 \%)$ have relapsed in this study, consisting of nodal recurrence in $17.6 \%$, hematogenous recurrence in $11.8 \%$, and peritoneal dissemination in $7.8 \%$ of patients, compared with a total of $30.6 \%$ of patients who developed recurrences with adjuvant S-1 monotherapy after D2 gastrectomy in the ACTS-GC trial with the peritoneum (14.6\%), hematogenous sites (11.5\%), and lymph nodes $(5.7 \%)$ as common sites of relapse [11]. In addition, S-1 plus docetaxel has recently shown superior survival to S-1 monotherapy in advanced gastric cancer patients with nonmeasurable lesions [17]. These findings suggest that postoperative adjuvant chemotherapy with S-1 plus docetaxel followed by S-1 monotherapy for up to 1 year after surgery is favorable for patients who are likely to relapse in the peritoneum, i.e., patients with linitis plastica.

In this study, 3-year OS as well as 3-year DFS was evaluated. An extremely strong correlation between DFS and OS was demonstrated by the GASTRIC group metaanalysis of 17 adjuvant trials after curative resection of gastric cancer [21]. In recent large clinical trials of adjuvant chemotherapy after curative D2 gastrectomy, 3-year RFS or 3-year DFS has been evaluated as surrogate measures of 5-year OS $[10,20]$, and it has been proven to become the primary endpoint for potentially curable gastric cancer, reconfirming the strong concordance of 3-year RFS with 5-year OS [11].

In conclusion, this 3-year follow-up study may suggest the efficacy of postoperative adjuvant chemotherapy with 
four cycles of S-1 plus docetaxel followed by S-1 monotherapy for up to 1 year after D2 gastrectomy in patients with stage IIIA gastric cancer, while further studies for stage IIIB patients are needed. However, this study is a small-scale phase II study, not large enough to draw a definite conclusion on the survival benefit of combined adjuvant chemotherapy with S-1 plus docetaxel over S-1 monotherapy for stage III gastric cancer. Superior feasibility of this combined regimen in the adjuvant setting, and comparable OS and DFS in both regimens as shown in Table 2, warrant future phase III trial (S-1 plus docetaxel versus S-1 monotherapy) to identify the optimal adjuvant chemotherapy regimen for stage III gastric cancer.

Conflict of interest The authors declare no potential conflicts of interest.

\section{References}

1. Hermans J, Bonenkamp JJ, Boon MC, Bunt AMG, Ohyama S, Sasako M, et al. Adjuvant therapy after curative resection for gastric cancer: meta-analysis of randomized trials. J Clin Oncol. 1993;11:1441-7.

2. Pignon J, Decreux M, Rougier P. Meta-analysis of adjuvant chemotherapy in gastric cancer: a critical reappraisal. J Clin Oncol. 1994;12:877-8.

3. Earle CC, Maroun JA. Adjuvant chemotherapy after curative resection for gastric cancer in non-Asian patients: revisiting a meta-analysis of randomised trials. Eur J Cancer. 1999;35: 1059-64.

4. Mari E, Floriani I, Tinazzi A, Buda A, Belfiglio M, Valentini M, et al. Efficacy of adjuvant chemotherapy after curative resection for gastric cancer: a meta-analysis of published randomised trials. Ann Oncol. 2000;11:837-43.

5. Janunger K, Hafstrom L, Nygren P, Glimelius B. A systematic overview of chemotherapy effects in gastric cancer. Acta Oncol. 2001;40:309-26.

6. Panzini I, Gianni L, Fattori P, Tassinari D, Imola M, Fabbri P, et al. Adjuvant chemotherapy in gastric cancer: a meta-analysis of randomized trials and a comparison with previous meta-analyses. Tumori. 2002;88:21-7.

7. GASTRIC (Global Advanced/Adjuvant Stomach Tumor Research International Collaboration) Group, Paoletti X, Oba K, Burzykowski T, Michiels S, Ohashi Y, Pignon JP, et al. Benefit of adjuvant chemotherapy for resectable gastric cancer: a metaanalysis. JAMA. 2010;303:729-37.

8. Macdonald JS, Smalley SR, Benedetti J, Hundahl SA, Estes NC, Stemmermann GN, et al. Chemoradiotherapy after surgery compared with surgery alone for adenocarcinoma of the stomach or gastroesophageal junction. N Engl J Med. 2001;345:725-30.
9. Cunningham D, Allum WH, Stenning SP, Thompson JN, Van de Velde CJ, Nicolson M, et al. Perioperative chemotherapy versus surgery alone for resectable gastroesophageal cancer. N Engl J Med. 2006;355:11-20.

10. Sakuramoto S, Sasako M, Yamaguchi T, Kinoshita T, Fujii M, Nashimoto A, et al. Adjuvant chemotherapy for gastric cancer with S-1, an oral fluoropyrimidine. N Engl J Med. 2007;357: 1810-20.

11. Sasako M, Sakuramoto S, Katai H, Kinoshita T, Furukawa H, Yamaguchi T, et al. Five-year outcomes of a randomized phase III trial comparing adjuvant chemotherapy with S-1 versus surgery alone in stage II or III gastric cancer. J Clin Oncol. 2011;29:4387-93.

12. Takahari D, Hamaguchi T, Yoshimura K, Katai H, Ito S, Fuse N, et al. Feasibility study of adjuvant chemotherapy with S-1 plus cisplatin for gastric cancer. Cancer Chemother Pharmacol. 2011;67:1423-8.

13. Tamura S, Fujitani K, Kimura Y, Tsuji T, Matsuyama J, Iijima S, et al. Phase II feasibility study of adjuvant S-1 plus docetaxel for stage III gastric cancer patients after curative D2 gastrectomy. Oncology. 2011;80:296-300.

14. Japanese Gastric Cancer Association. Japanese Classification of Gastric Carcinoma-2nd English Edition. Gastric Cancer. 1998;1: $10-24$.

15. Koizumi W, Narahara H, Hara T, Takagane A, Akiya T, Takagi $\mathrm{M}$, et al. S-1 plus cisplatin versus S-1 alone for first-line treatment of advanced gastric cancer (SPIRITS trial): a phase III trial. Lancet Oncol. 2008;9:215-21.

16. Van Cutsem E, Moiseyenko VM, Tjulandin S, Majlis A, Constenla M, Boni C, et al. Phase III study of docetaxel and cisplatin plus fluorouracil compared with cisplatin and fluorouracil as firstline therapy for advanced gastric cancer: a report of the V325 Study Group. J Clin Oncol. 2006;24:4991-7.

17. Kim YH, Koizumi W, Lee KH, Kishimoto T, Chung HC, Hara T, et al. Randomized phase III study of S-1 alone versus S-1 + docetaxel in the treatment for advanced gastric cancer: the START trial. J Clin Oncol 2011;29(suppl 4):abstr 7.

18. Sasako M, Yamaguchi T, Kinoshita T, Fujii M, Nashimoto A, Furukawa $\mathrm{H}$, et al. Randomized phase III trial comparing S-1 monotherapy versus surgery alone for stage II/III gastric cancer patients after curative D2 gastrectomy (ACTS-GC study). ASCOGI 2007; abstr 8.

19. Sobin LH, Wittekind $\mathrm{CH}$. International Union Against Cancer (UICC) TNM classification of malignant tumours, 6th edn. New York; Wiley-Blackwell; 2002.

20. Bang YJ, Kim YW, Yang HK, Chung HC, Park YK, Lee KH, et al. Adjuvant capecitabine and oxaliplatin for gastric cancer after D2 gastrectomy (CLASSIC): a phase 3 open-label, randomised controlled trial. Lancet. 2012;379:315-21.

21. Burzykowski T, Bang Y, on behalf of the GASTRIC project. Disease-free survival as a surrogate endpoint for overall survival in an adjuvant trial of curatively resected stomach cancer using individual patient data meta-analysis. J Clin Oncol 2009; 27(15s), abstr 4517 